\title{
Ground truth energies for hierarchies of segmentations
}

\author{
B. Ravi Kiran, Jean Serra \\ Université Paris-Est, Laboratoire d'Informatique Gaspard-Monge, A3SI, ESIEE \\ $\{j$.serra, kiranr, \}@esiee.fr
}

\begin{abstract}
In evaluating a hierarchy of segmentations $\mathrm{H}$ of an image by ground truth $\mathrm{G}$, which can be partitions of the space or sets, we look for the optimal partition in $\mathrm{H}$ that "fits" G best. Two energies on partial partitions express the proximity from $\mathrm{H}$ to $\mathrm{G}$, and $\mathrm{G}$ to $\mathrm{H}$. They derive from a local version of the Hausdorff distance. Then the problem amounts to finding the cut of the hierarchy which minimizes the said energy. This cuts provide global similarity measures of precision and recall. This allows to contrast two input hierarchies with respect to the $\mathrm{G}$, and also to describe how to compose energies from different ground truths. Results are demonstrated over the Berkeley database.
\end{abstract}

\section{Introduction}

Classically, the evaluation of a segmentation w.r.t. a ground truth is viewed as a problem of comparing two partitions of the space $E$. There are various metrics proposed which are described well surveyed in [4]. The thesis [1] provides refinement tolerant based errors, Local and Global consistency errors(LCE, GCE), due to differences in rendering the ground truth by different human experts. [4] proposes a local region based measure, the segmentation covering, which is the ratio of intersection of 2 classes over the union of their supports, weighted by the relative size of the class w.r.t the input image.

This method is also used to evaluate classes, regions and full partitions of the hierarchy which correspond to the threshold of the Ultrametric contour Map(UCM). As pointed out in [5] the merging order is not the only "cut" in a hierarchy of partitions. The total number of cuts possible consists of the set of partitions formed by the power set of the classes of leaves in the finest partition in a hierarchy.

On the subject of evaluating hierarchies of segmentations there is the work of J. Pont-Tuset and F. Marques [5] closest to this subject of the paper. They determine the upper bound on the correspondences between a ground truth partition and all partitions in a input hierarchy. The comparison performs a global match correspondence between all contours in the hierarchy with respect to the Ground truth partition contours. This thus involves a combinatorial optimization problem, since one must choose a set of contours at various levels and having minimal distance from the ground truth. Indeed, the upper bound 
introduced in [5] is nothing but the optimal cut in the sense of [8], i.e. the cut which minimizes a given energy, and whose computation is extremely easy as soon as the energy is $h$-increasing [6]. [9] also propose a local optimization which depends on the number of classes in the cut in a hierarchy with respect to the ground truth segmentation.

The last remark orients us towards the convenient classes of energies acting on hierarchies, as studied in [8]. These energies will be addressed to evaluate hierarchies, a question which covers three aspects:

1. Given a hierarchy $H$ of segmentations of an image $I$, and a ground truth partition $G$, how to find a local and a global measures of proximity of the quality of $H$ relatively to $G$ and vice versa, $G$ relative to $H$ ? By local here, we mean a space map of the quality. In fact, we will see that this involves two reciprocal notions. Note that $G$ may be, or not, a contour, but models a drawing by lines and points $(G=\partial G)$.

2. When several humans provide several ground truths, how to compose information from multiple ground truth sets $G_{i}$ ? What to do in particular when each drawing concerns a limited zone of the space, which varies with the human/expert?

3. Finally how to evaluate globally the proximities any two given different hierarchies $H_{1}, H_{2}$, with respect to a given common ground truth $G$.

To summarise symbolically: $1 . H \rightarrow G$ and $G \rightarrow H, 2$. $H \rightarrow G_{i}$ and vice versa, where $G_{i}$ refers to a set of ground truths indexed by $i, 3 . H_{j} \rightarrow G$, where $H_{i}$ refers to different input hierarchies, indexed by $j$.

After a brief recall of optimal cuts and the optimization framework, the above three problems will be successively tackled: the first two by optimal cuts, and the third one by means of global similarity measure defined on the saliency function representing the hierarchy. For the sake of pedagogy, we demonstrate on one image, namely the $\mathrm{n}^{\circ} 25098$ of Berkeley data base, and on the two ground truths depicted in Fig.1, though results are available over available over all images in the database shortly.

\section{Reminders}

\subsection{Hierarchy and Saliency}

We start from the definitions used in [8] where,a hierarchy $H$ is a finite chain of partitions $\pi_{i}$, i.e.

$$
H=\left\{\pi_{i}, 0 \leq i \leq n \mid i \leq k \leq n \Rightarrow \pi_{i} \leq \pi_{k}\right\},
$$

The lowest level $\pi_{1}$ is called "the leaves", and the highest one is $E$ itself. An energy $\omega$ is associated with each partial partition of $E$. If $\mathcal{D}(E)$ designates the set of the partial partitions $E$, then $\omega: \mathcal{D}(E) \rightarrow \mathbb{R}^{+}$. Let $\pi_{1}$ and $\pi_{2}$ be two partial partitions of same support, and $\pi$ be a partial partition disjoint from $\pi_{1}$ and 


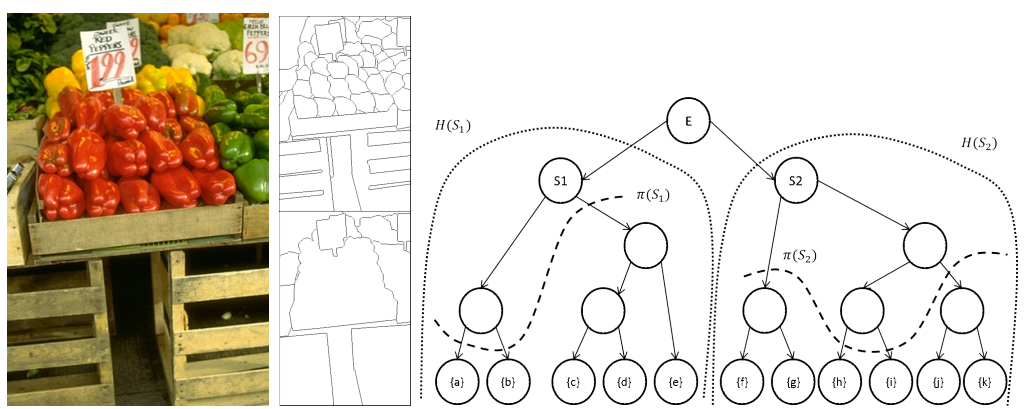

Fig. 1. left: 25098 image from BSD database and 2 of its ground truths GT2 and GT7, right: A hierarchy $H$ with undulating cuts shown $\pi\left(S_{1}\right)$ and $\pi\left(S_{2}\right)$

$\pi_{2}$. An energy $\omega$ on $\mathcal{D}(E)$ is said to be hierarchically increasing, or h-increasing when we have

$$
\omega\left(\pi_{1}\right) \leq \omega\left(\pi_{2}\right) \quad \Rightarrow \quad \omega\left(\pi_{1} \sqcup \pi\right) \leq \omega\left(\pi_{2} \sqcup \pi\right) .
$$

This condition is necessary and sufficient for obtaining the cut(s) which minimize $\omega$, by running only once through the classes of $H$ in an ascending order. This provides for a dynamic program that only performs a local comparison between a parent class and a composition of its children classes in the hierarchy. The most popular representation of a hierarchy is the dendrogram, shown in (figure). The advantage of this representation is it makes explicit the parent-child relation. Another useful representation, more compact, is the saliency map. It consists in a weighted version of all the edges separating the leaves. Each threshold of the saliency map results in an horizontal cut in the hierarchy. Intuitively, the saliency map is a function that helps visualize the different prominent partitions in the hierarchy.

\subsection{Hausdorff distance}

Most of the supervised evaluations of hierarchies, including the present one, and also [2], [4], and [5], derive from the intuition of the Hausdorff distance, in various critical manners. Let us briefly recall this background.

In a metric space $E$ of distance $d$ we aim to match the support $S(\pi)$ of a bounded partial partition $\pi$ with a set $G$ of points and lines, considered as a ground truth drawing. The smallest isotropic dilation of $G$ that covers the contour $S(\pi)$ has a radius

$$
\rho_{G}=\inf \{\rho \mid G \oplus \rho B \supseteq S(\pi)\},
$$

where $\rho B$ is the disc of radius $\rho$ centred at the origin. One can interpret $\rho_{G}$ as the "energy" required for reaching $\partial S$ from the ground truth $G$. In the same 
way, the dual covering is given by the radius $\rho_{A}$

$$
\rho_{A}=\inf \{\rho \mid S(\pi) \oplus \rho B \supseteq G\} .
$$

By introducing the so called distance function $d(x, Z)$ from point $x$ to the fixed set $Z$, i.e.

$$
d(x, Z)=\inf \{d(x, z), z \in Z\} \quad x \in E
$$

we see that

$$
\rho_{G}=\sup \{d(x, G), x \in S(\pi)\} \quad \text { and } \quad \rho_{A}=\sup \{d(x, S(\pi)), x \in G\},
$$

an interpretation which connects the distance function with the partial order on sets by inclusion. In Rel.(6) the value $\rho_{G}$ (resp. $\rho_{A}$ ) is the maximal distance from a point of $\partial S$ to $G$ (resp. of $G$ to $\partial S$ ). The first one, $\rho_{G}$, indicates how precise is $S$ w.r.t. the ground truth, the second one, $\rho_{A}$, how representative is this ground truth. In indexation, these two numbers are respectively named precision and recall. The symmetric expression $\rho=\max \left\{\rho_{G}, \rho_{A}\right\}$ is the well known Hausdorff distance

Hausdorff distance is lacking of finesse because it is a global notion, and of robustness because it uses suprema. If we could define a local equivalent, associated with each class $T$ of $\pi$, and no longer with the whole $S(\pi)$ itself, then the regions with a good fit would be treated separately from the others. And in addition, if this equivalent was $h$-increasing, then it would provide an energy for calculating easily the associated optimal cut [8], i.e. the smallest upper bound of all cuts of the hierarchy, in the wording of [5]).

\section{Ground truth energy by local Hausdorff dissimilarity}

In what follows, "best cut", or "optimal cut" must be understood in the sense of "best fitting cut", i.e. the cut which minimizes a given energy of proximity with the ground truth $G$. It is usually not a criterion of best visual quality.

Precision energy We now focus on the classes $\left\{T_{i}\right\}$ whose concatenation $T_{i} \sqcup T_{2} \ldots \sqcup T_{k}$ generates $\pi$. The $\left\{T_{i}\right\}$ are said to be the sons of father $S$. Consider the class $T_{i}$ of the partition $\pi$. The smallest dilate $G \oplus \rho B$ that covers $T_{i}$ has a radius

$$
\omega_{G}\left(T_{i}\right)=\inf \left\{\rho \mid G \oplus \rho B \supseteq T_{i}\right\} .
$$

By taking the supremum of all $\omega_{G}(S)$ we find the above value $\rho_{G}$ of Rel.(3):

$$
\rho_{G}=\bigvee\left\{\omega_{G}(S), S \sqsubseteq \pi_{A}\right\} .
$$

This shows the soundness of $\omega_{G}$. But a problem arises when we want to extend it from sets to the partial partitions $\mathcal{D}(E)$ of $E$ by some law of composition between 


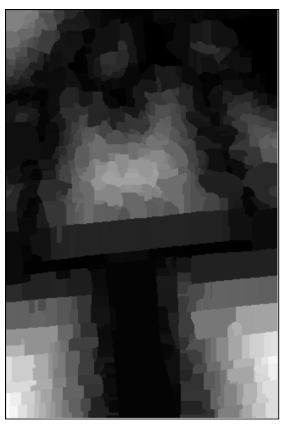

a)

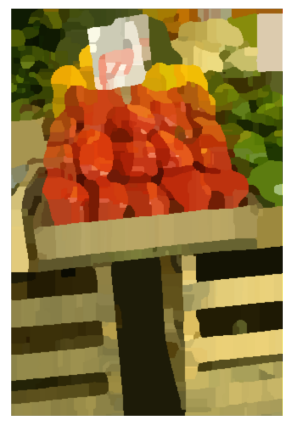

b)

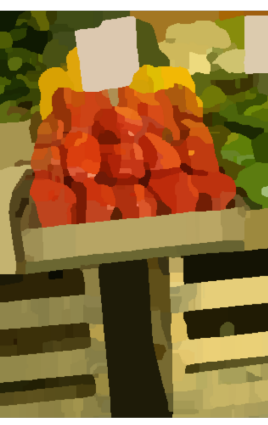

c)

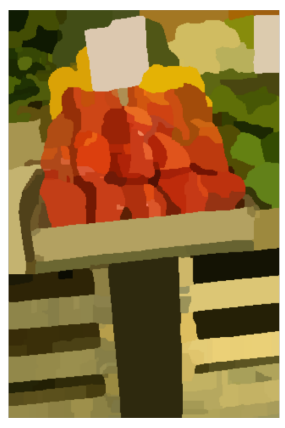

d)

Fig. 2. a) Distribution of the energy $\omega_{G}$ of the leaves classes; b) c) and d) optimal cuts for $\lambda=0 ; 10$; and 20 .

the $T_{i}$. When the chosen energy is $h$-increasing, which will always be the case here, finding optimal cuts in hierarchies amounts to compare the partition energies of fathers and sons [8]. If we compose the energies of the sons by supremum, then we trivially always find $\omega_{G}(\pi)=\omega_{G}(S)$. If we compose by infimum, we have $\omega_{G}(\pi)=\omega_{G}(S)$ when the $\omega_{G}\left(T_{i}\right)$ all identical, and $\omega_{G}(\pi)<\omega_{G}(S)$ when not. And if we compose the energies of the sons by averaging, we obtain again $\omega_{G}(\pi)<\omega_{G}(S)$. Therefore, in all cases, we arrive to an optimal cut which can only be at the lowest level of hierarchy $H$, i.e. the leaves, or at the highest one, i.e. the space $E$ itself.

For being more informative, we can introduce a trade off based on mutual comparisons of the energies of the sons. An easy way consists in adding a quantizer $\lambda$ in the composition by infimum, so that

$$
\omega_{G}(\pi)=\omega_{G}\left(T_{i} \sqcup T_{2} \ldots \sqcup T_{k}\right)=\inf \left\{\omega_{G}\left(T_{i}\right)\right\}+\lambda .
$$

As this new energy is $h$-increasing, the optimal cut is reached in one pass by comparing the respective energies of sons and fathers [8]. As $\omega_{G}(S)=$ $\sup \left\{\omega_{G}\left(T_{i}\right)\right\}$, we have $\omega_{G}(\pi)<\omega_{G}(S)$ iff $\lambda<\sup \left\{\omega_{G}\left(T_{i}\right)\right\}-\inf \left\{\omega_{G}\left(T_{i}\right)\right\}$. The father replaces the sons when the latter are sufficiently "identical" , i.e. with energy variation $\leq \lambda$. For each value of $\lambda$ we thus obtain the cut which minimizes the distances to the ground truth $G$, i.e. the smallest upper bound of all cuts, in the sense [5]. For $\lambda=0$ we find the leaves partition, and as $\lambda$ increases, the similar sons w.r.t. their distance to $G$ are progressively clustered, as shown in Fig. ?? of the leaves classes for the ground truth $G T 2$; b) c) and d) optimal cuts for $\lambda=0 ; 10$; and 20 .

Recall energy The number $\omega_{G}(S)$ informs us about those points of $\partial S$ close enough to $G$, but not on those of $G$ close to $\partial S$. We cannot take, here, the 
dual form of the $\omega_{G}(S)$ of Rel.(7), as we did before with the global Hausdorff distance. Such a dual energy would be

$$
\omega_{G}^{\prime}(S)=\inf \{\rho \mid S \oplus \rho B \supseteq G\},
$$

a quantity which risks to be extremely large, for the drawing $G$ may spread over the whole space, whereas class $S$ is locally implanted. Fortunately, when dealing with $h$-increasing energies, one is less interested in the actual values of the energies than by their increments between fathers and sons. Now, when a point of $G$ is outside class $S$, then its distance to $S$ is the same as the max of the distances to the sons $T_{i}$ of $S$ :

$$
x \in G \cap S^{c} \Rightarrow d(x, S)=d(x, \partial S)=\bigvee d\left(x, T_{i}\right)=\bigvee d\left(x, \partial T_{i}\right),
$$

so that the part of $G$ exterior to $S$ is not significant. For the sake of comparison, it thus suffices to focus only on the distances involved in the covering of $G \cap S$ by dilations of $\partial S$ on the one hand, and on those of $\partial T_{i}$ on the other hand. Then the energy $\omega_{G}^{\prime}$ of Rel.(10) has to be replaced by the more appropriate one

$$
\theta_{G}(S)=\inf \{\rho \mid S \oplus \rho B \supseteq G \cap S\} .
$$

When $S$ spans all classes of a partition $\pi_{A}$, then the supremum of all $\theta_{G}(S)$ gives the value $\rho_{A}$ of Rel.(4)

$$
\rho_{A}=\bigvee\left\{\theta_{G}(S), S \sqsubseteq \pi\right\},
$$

and the (global) Hausdorff distance $\rho$ between $\pi$ and $G$ turns out to be the double supremum,

$$
\rho=\bigvee\left\{\left\{\omega_{G}(S) \bigvee\left\{\theta_{G}(S)\right\}, S \sqsubseteq \pi\right\}\right.
$$

It remains to verify that $\theta_{G}$ is $h$-increasing.

Proposition 1. Given a ground truth set $G$, the extension of the energy $\theta_{G}$ of Rel.(12) to partial partitions by $\vee$ composition is h-increasing.

Proof. Let $\pi\left(S_{1}\right)$ and $\pi^{\prime}\left(S_{1}\right)$ be two partial partitions of set $S_{1}$, with

$$
\theta_{G}\left(\pi\left(S_{1}\right)\right)=\bigvee\left\{\theta_{G}\left(T_{i}\right), T_{i} \sqsubseteq S\right\} \leq \theta_{G}\left(\pi^{\prime}\left(S_{1}\right)\right)=\bigvee\left\{\theta_{G}\left(T_{i}^{\prime}\right), T_{i}^{\prime} \sqsubseteq S_{1}^{\prime}\right\}
$$

Consider a partial partition $\pi\left(S_{2}\right)$, where $S_{2} \subseteq S_{1}^{c}$. By taking the supremum of each member of inequality (15) with $\bigvee\left\{\theta_{G}\left(X_{j}\right), X_{j} \sqsubseteq S_{2}\right\}$ one does not change the sense of the inequality, which becomes

$$
\theta_{G}\left(\pi\left(S_{1}\right) \sqcup \pi\left(S_{2}\right)\right) \leq \theta_{G}\left(\pi^{\prime}\left(S_{1}\right) \sqcup \pi\left(S_{2}\right)\right),
$$

which achieves the proof.

Note that when $G \cap S=\varnothing$, then $\theta_{G}(S)=0$. 


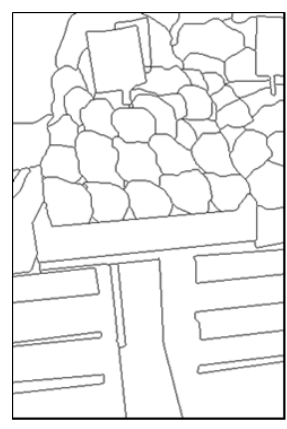

a)

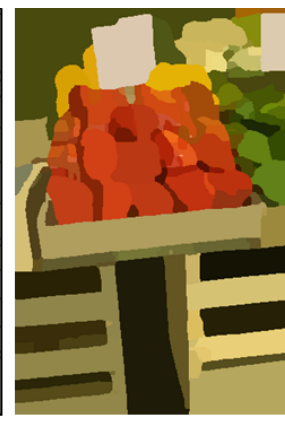

b)

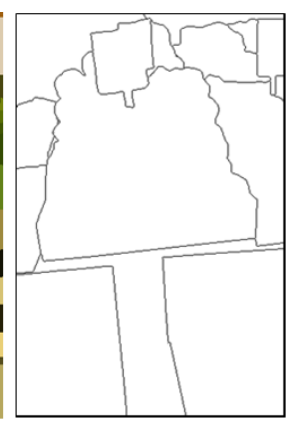

c)

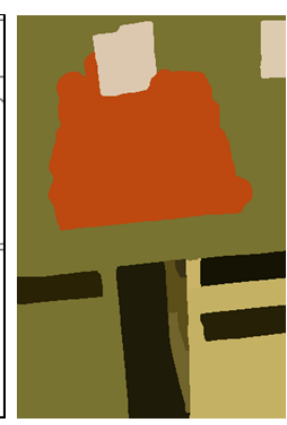

d)

Fig. 3. a) and c), ground truth $G T 2$ and $G T 7$; b) and d) energies $\omega_{G}+\theta_{G}$, for $G=G T 2$ and $G=G T 7$.

Composition of $\omega_{G}(S)$ and $\theta_{G}(S)$. The composition of the energies happens with respect to a single ground truth, or to several ones. In the first case, one can wonder if preferable not to combine $\omega_{G}$ and $\theta_{G}$ so that they can provide two separated maps for the precision and for the recall. The two associated overall values may be presented in a 2-D graphic as proposed in [3]. We can also take for final energy either $\max \left(\omega_{G}, \theta_{G}\right)$, or sum $\omega_{G}+\theta_{G}$, they are both $h$-increasing. On the example of the "peppers", and for two different ground truths, one obtains the results depicted in Fig.3

In case of multiple ground truths, the usual techniques proposed in literature are additive. Formally speaking, why not? Putting $\omega_{G}=\sum \omega_{G_{i}}$ yields an $h$ increasing energy, hence a best cut (which is, of course different from the sum of the best cuts of the various $G_{i}$ ). The implicit assumption here is that all ground truths are more or less similar.

But one can also encounter drawings $G_{i}$ that focus on different regions of the scene. Then if we take the sum, each part of the space risks to be penalized because if is far from one drawing, at least.

For the situation depicted in Fig.4, the energies first two best cuts are given by $\sup \left\{\omega_{G}, \theta_{G}\right\}$ and the third one by taking $\inf \left\{\sup \left\{\left\{\omega_{G_{1}}, \theta_{G_{1}}\right\}, \sup \left\{\left\{\omega_{G_{2}}, \theta_{G_{2}}\right\}\right\}\right.\right.$. When point $x \in E$ is farther from $G_{1}$ than from $G_{2}$ then the $G_{1}$ energy is not taken into account.

\section{Other energies}

Conditional energy The two energies $\omega_{G}(S)$ and $\theta_{G}(S)$ of Rel. (12) and (9) have been chosen because of their geometrical meanings, but they are far for being the only possible ones. It is iindeed easy to build an energy which fits the features one wants to emphazise. Suppose for example that we decide that the number of classes $n$ of the ground truth is a cruxial feature. Then when applying energy $\omega_{G}$ we can condition the ascending pass which generates the best cut to stop as 


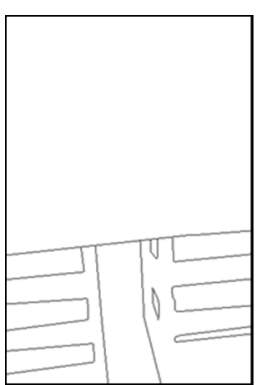

a)

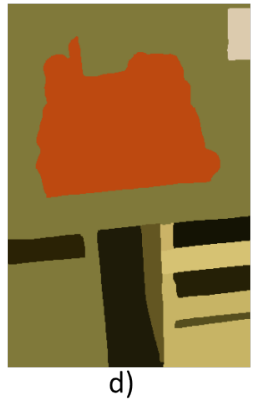

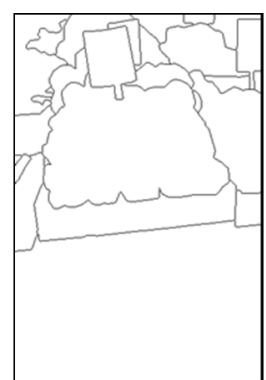

b)

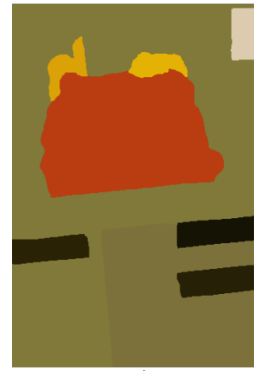

e)

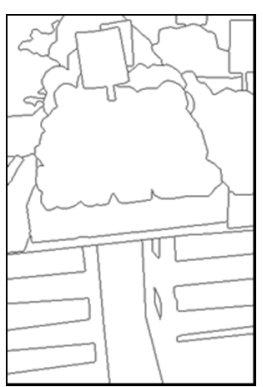

c)

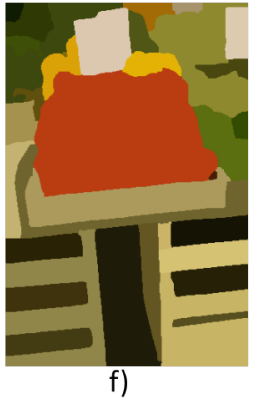

Fig. 4. a),b), and c) two ground truths and their union; d),e), and f) the coresponding optimal cuts.

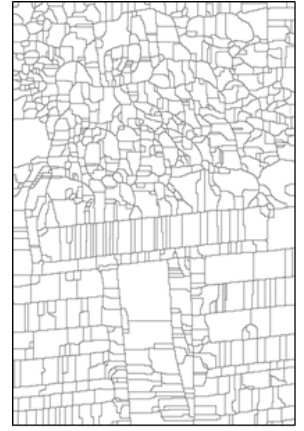

a)

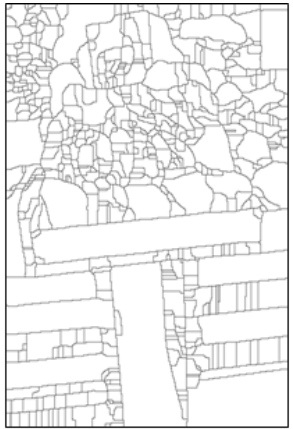

b)

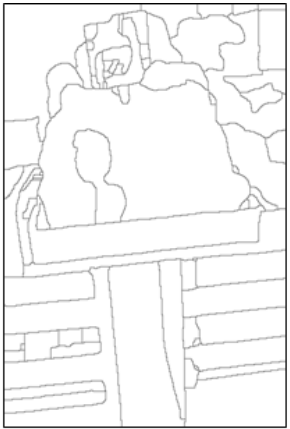

c)

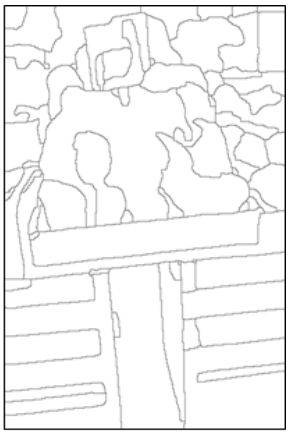

d)

Fig. 5. a) Leaves of hierarchy b), c) and d) Conditional best cuts for $\lambda=0,10$ and 80 . 
soon as the number $n$ of classes is reached. Fig. 5 depicts the best cuts w.r.t. $\omega_{G}$. when the parameter $\lambda$ of Eq.(9) equals 0,10, and 80, and when the ground truth is GT7, which has 87 classes. For $\lambda=0$, we do not obtain the leaves partition, because the classes with an equal energy have been clustered, as pointed out previously. In Fig. 5c) and d), but not in Fig. 5b), one arrives to 87 classes before the end of the climbing algorithm. This explains why the two partitions are not comparable.

Local linear dissimilarity Another variant consists in replacing the supremum that appears in Rel.(6) by a $L^{p}$ sum, which gives less importance to the farthest zones. A similar approach has been successfully used by L. Gorelick et Al. [12] in regional line-search cuts. Among the $L^{p}$ integrals, the one which weakens the most the weights of the farthest zones is obtained for $p=1$. Therefore we take for precision energy $\widetilde{\omega}_{G}(S)$ the integral of distance function $g(x)$ of $G$ along the contour $\partial S$ and for recall energy $\widetilde{\theta}_{G}(S)$ the integral of the distance function $g(x, \partial S)$ of $S$ on $G \cap S$ :

$$
\widetilde{\omega}_{G}(S)=\frac{1}{\partial S} \int_{\partial S} g(x) d x \quad \widetilde{\theta}_{G}(S)=\frac{1}{G \cap S} \int_{G \cap S} g(x, \partial S) d x
$$

The two functionals $\widetilde{\omega}_{G}$ and $\widetilde{\theta}_{G}$ are extended from classes to partial partitions by addition, since they both involve integrals, and one easily checks that the two energies are $h$-increasing. The higher $\widetilde{\omega}_{G}(S),\left(\operatorname{resp} . \widetilde{\theta}_{G}(S)\right)$, the farther $S$ is from $G$ (resp. $G$ is from $S$ ). In case of a ground truth given by $k$ drawings, one just sums up the $k$ energies $\widetilde{\omega}_{G}$ and $\widetilde{\theta}_{G}$.

\section{Global measures of precision and recall for hierarchies}

Following from the local measures in (17) which are integrals of the distance function associated with each class, we propose here a global similarity measures for a hierarchy. Two global measures of precision and recall for a given hierarchy $H$ of segmentations with respect to an input ground truth partition $G$. The measure now is not between 2 partitions any more and deals with the global similarity between hierarchies of partitions $H$ and a single partition $G$. The representative functions we are going to use for the global measures are: $s$ the saliency and $g$ the distance function, the set $S_{i}$ saliency map threshold at an index $i$.

$$
P=\sum_{i=0}^{1} \frac{i}{N} \frac{\int_{x \in \epsilon\left(S_{i}\right)}(1-g(x)) \cdot S_{i}(x) \mathrm{d} x}{\left|S_{i}\right|} \quad R=\sum_{i=0}^{1} \frac{i}{N} \frac{\int_{x \in G}\left(1-g_{S_{i}}(x)\right) \mathrm{d} x}{|G|}
$$

The integral calculates the similarity between partition $S_{i}$ produced by thresholding the saliency $s$ at $i$ and the ground truth partition $G$ by integrating the inverse distance function $1-g$ under the binary function $S_{i}$. Also the sense of the hierarchy is such that $s_{i+1} \subset s_{i}$ which represents that partition at a higher level in the hierarchy has fewer contours than the one below to respect 
the inclusion order. Each integral is weighted by the relative rank of the partition within the hierarchy $H$. This is done by weight it by ratio of threshold index $i$ and the total number of levels in the hierarchy $N$ as shown in equation(18).

Similarly a global precision value for the contours of the partitions in the hierarchy can be calculated by integrating the distance functions $g_{S_{i}}$ of partition $S_{i}$ under the ground truth partition $G$. These integrals are normalized with respect to each image support by dividing by the size of the image.

\subsection{Proximity between hierarchies}

The integrals in equation (18) is between a partition $G$ (ground truth) and a hierarchy $H$. The same can be extended to measure the proximities between two hierarchies of partitions. Given two hierarchies of partitions, $H_{1}, H_{2}$, with $\mathrm{N}$ and $\mathrm{M}$ number of levels, and partitions indexed by $i$ and $j$ respectively,

$$
\begin{aligned}
& \phi_{12}=\sum_{j \in[1, M]} \sum_{i \in[1, N]} \frac{\int_{x \in \epsilon\left(\pi_{i}\right)}\left(1-g_{\pi_{j}}(x)\right) \cdot \pi_{i}(x) \mathrm{d} x}{\left|\pi_{i}\right|} \\
& \phi_{21}=\sum_{i \in[1, N]} \sum_{j \in[1, M]} \frac{\int_{x \in \epsilon\left(\pi_{j}\right)}\left(1-g_{\pi_{i}}(x)\right) \cdot \pi_{j}(x) \mathrm{d} x}{\left|\pi_{j}\right|}
\end{aligned}
$$

where $g_{\pi_{i}}$ is the distance function of the partition $\pi_{i}$.

The measure lacks the similarity measures across partitions which are not horizontal cuts, but generally cuts from the two hierarchies. This becomes again a combinatorial problem. The refinement of cuts $\pi_{i}$ from an input hierarchy $H_{1}$ would have a value of the distance function $g_{\pi_{j}}$ which decreases on average till the point where the two partitions nearly fit and the integral starts increasing again.

\section{Results}

To demonstrate the inputs of the optimization, we show the 2 ground truths used (GT2 and GT4 from the list for image 239096), we show the distribution of ground truth energies(radii) at different thresholds of the saliency. The gray level corresponds to the radius of dilations $\omega_{G}$ and $\theta_{G}$ in Figure 7 . for image 239096. Their optimal cuts based on the haussdorf energy corresponding to the supremum of the two radii $\omega_{G}$ and $\theta_{G}$ are shown in Figure 8 . We observe that the optimization introduces small parasite classes which are chosen since the children or always more optimal than the parent in certain symmetries.

We evaluate the global measure on 3 hierarchies Arbelaez, Cousty and random hierarchies (generated by merging classes of the leaves partition randomly) for the 2 hierarchies produced from random permutation matrices used as distance functions as explained in the previous section. We evaluate all 7 ground truths w.r.t to the 3 input hierarchies, producing a table (9) of global measures. The $\mathrm{P}$ and $\mathrm{R}$ measures are averaged over the 7 ground truths available for 25098 image. 

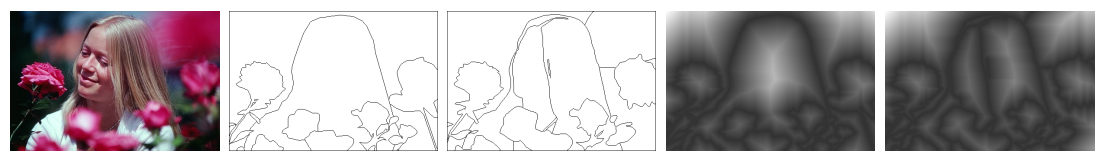

Fig. 6. Input Image 2390986, Ground truths GT2 and GT4, and their distance functions
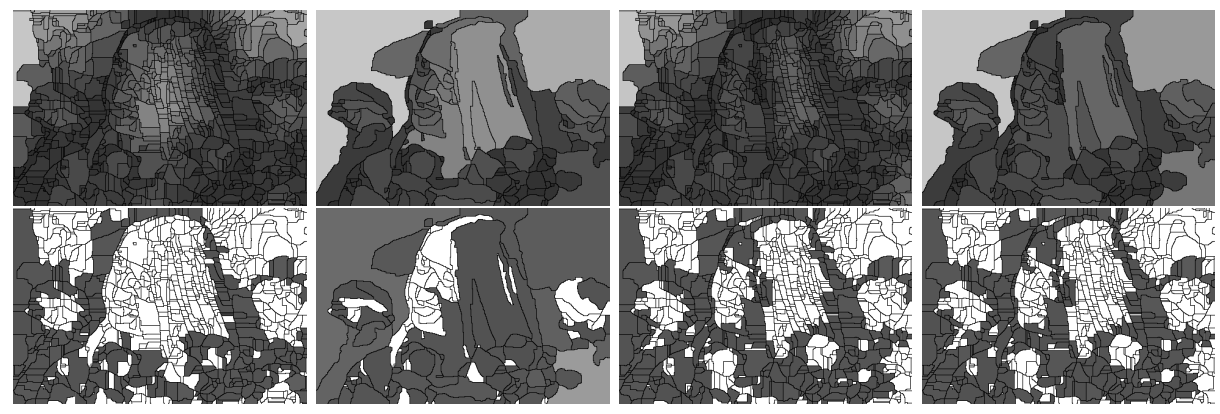

Fig. 7. The distribution of $\omega_{G}$ (top) for threshold of the (UCM) at 0 (leaves), 0.1 , for ground truth GT 2(two images on the left) and GT 4(right), the image is contrasted to see the low level values clearer. Same for $\theta_{G}$ on the bottom line
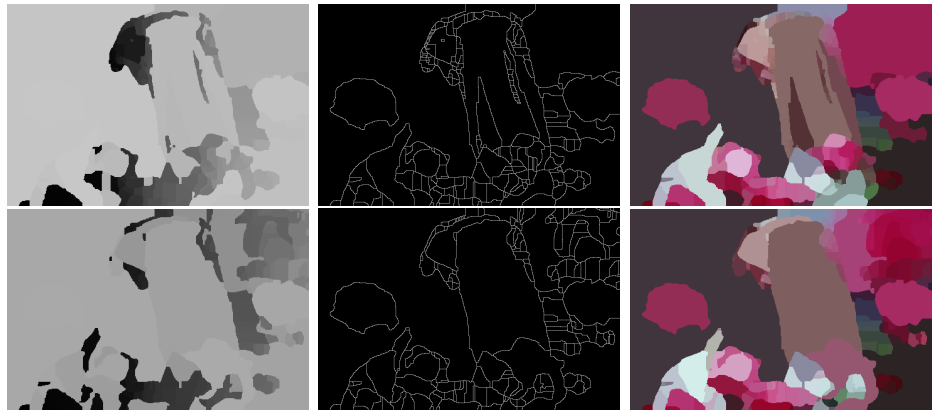

Fig. 8. The Energy distribution for the optimal cut by $\vee\left(\omega_{G}, \theta_{G},\right)$ The partition and the image

\begin{tabular}{|l|l|l|l|l|}
\hline $\begin{array}{l}\text { Image } \\
25098\end{array}$ & UCM & Cousty & $\begin{array}{l}\text { Random } \\
\text { UCM }\end{array}$ & $\begin{array}{l}\text { Random } \\
\text { Cousty }\end{array}$ \\
\hline $\mathrm{P}$ & 4.4 & 0.27 & 0.13 & 0.09 \\
\hline $\mathrm{R}$ & 3.9 & 0.28 & 0.16 & 0.10 \\
\hline
\end{tabular}

Fig. 9. Integrals from equation(18) Expressed per 1000 pixels in the image 


\section{Conclusion}

A method for comparing a hierarchy of partitions $H$ with one, or more, ground truth set $G$ was proposed. Two points of view were developed. The first one is based on the idea of associating two energies that express the proximity between $G$ and $H$. It was shown that several different criteria, and several laws of composition of the partition's classes lead to emphasize different aspects of the hierarchy. The same approach permits also to combine different ground truths associated with different zones of the space. Finally a global similarity measure is used to evaluate the proximity of hierarchies of partitions and a ground truth partition. Future work would consist in using other image feature based energies and studying the law of compositions.

\section{References}

1. Martin, D.R, An Empirical Approach to Grouping and Segmentation, PhD Thesis, EECS Department, University of California, Berkeley, 2003, Number = UCB/CSD03-1268

2. Arbeláez, P. Une approche mtrique pour la segmentation d'images, Phd thesis, Univ.of Paris Dauphine, Nov. 2005

3. Arbeláez, P., Cohen L., Constrained Image Segmentation from Hierarchical Boundaries CVPR, (2008)

4. Arbeláez, P., Maire, M., Fowlkes, C., Malik, J.: Contour Detection and Hierarchical Image Segmentation. IEEE PAMI 33 (2011)

5. Jordi Pont-Tuset and Ferran Marqués, Supervised Assessment of Segmentation Hierarchies - In ECCV 20125-46

6. Serra, J., Hierarchy and Optima, in DGCI, LNCS 6007, Springer 2011, pp 35-46

7. Serra, J., Kiran, B.R. Climbing the pyramids CoRR abs/1204.5383 (2012).

8. Serra, J., Kiran, B. R., Cousty, J., Hierarchies and climbing energies, in CIARP 2012, L. Alvarez et al. (Eds.) LNCS 7441, (2012) 821-828.

9. Pont-Tuset J, Marqués F. Upper-bound assessment of the spatial accuracy of hierarchical region-based image representations. In: IEEE International Conference on Acoustics, Speech, and Signal Processing. 2012

10. Movahedi, V.Elder, J.H. Design and perceptual validation of performance measures for salient object segmentation. Computer Vision and Pattern Recognition Workshops (CVPRW), 2010 IEEE Computer Society Conference on, 49-56.

11. Cousty J. and Najman L. Incremental algorithm for hierarchical minimum spanning forests and saliency of watershed cuts, LNCS 6671 Springer, ISMM 2011

12. Gorelick L., Schmidt F. R., Boykov Y., Delong A., Ward A., Segmentation with non-linear regional constraints via line-search cuts, ECCV2012, LNCS 7572. 